\title{
Assessment of Early Initiation of Breastfeeding and Associated Factors Among Mothers in Benishangul Gumuz regional State, North West, Ethiopia: Community Based Cross-Sectional Study.2016
}

\author{
Yonas Deressa ${ }^{1 *}$, Girma Tadese ${ }^{2}$, Muluwas Amentie $^{3}$ \\ ${ }^{I}$ Department of Nursing, Pawe College of Health Sciences, Pawe, Ethiopia \\ ${ }^{2}$ Department of Research and Publication, Faculty of Health Sciences, Assosa University, Assosa, Ethiopia \\ ${ }^{3}$ Department of Public Health, Faculty of Health Sciences, Assosoa University, Assosa, Ethiopia
}

\begin{abstract}
Address for
Correspondance

Yonas Deressa,

$\underline{\text { Yonasderessa19 }}$

@.gmail.com
\end{abstract}

Received:

30.03.2017

Accepted:

28.05.2017

\begin{abstract}
Promoting early initiation of breastfeeding within 1 hour of birth and exclusive breastfeeding up to 6 months of age in early infancy is regarded as crucial components of child survival strategies that could avert up to $13 \%$ of deaths among children under 5 years of age. In addition, improved early initiation of breastfeeding appears to be a key in predicting neonatal mortality. Hence, this study aimed to assess prevalence of early initiation of breastfeeding and associated factors among mothers of children age less than two years northwest Ethiopia. Methods: Both quantitative and qualitative community-based cross-sectional study were conducted in seven woreda of Benishangul Gumuz Regional state on 590 infant paired mothers less than two years using simple random sampling. Data was coded, edited, entered into EPi-Info version 3.5.1 and analyzed by using SPSS version 20.0. Both descriptive and multivariable logistic regressions were used for data analysis. Results: A total of 770 women were participated with a response rate of $97.7 \%$. The prevalence of early initiation of breast feeding was 53.8\%. ANC follow up [AOR=2.02(1.07-3.92)], institutional delivery $[\mathrm{AOR}=12.18(8.29-17.98)]$, having adequate knowledge about breast feeding $[\mathrm{AOR}=1.9(1,001-2.23)]$, having adequate knowledge about early initiation of breast feeding $[\mathrm{AOR}=2.08(1.28-3.39)]$, were independently associated with early initiation of breast feeding practice. Conclusions: Early initiation of breast feeding is low as nearly half the mothers did not start breastfeeding with one hour after delivery and still lower than the national plan. ANC follow up, place of delivery, having adequate knowledge, were the contributing factors early initiation of breast feeding. Increasing maternal knowledge on early initiation of breast feeding by educating the mothers both at community and institutional levels and enhance adequate uterine contraction. (C) 2017 iGlobal Research and Publishing Foundation. All rights reserved.
\end{abstract}

Keywords Early Initiation of Breast Feeding; Infant and Young Child Feeding; Mothers; Ethiopia.

\section{INTRODUCTION}

Promoting early initiation of breastfeeding within 1 hour of birth and exclusive breastfeeding up to 6 months of age in early infancy are regarded as crucial components of child survival strategies that could avert up to $13 \%$ of deaths among children under 5 years of age $[1,2]$. In addition, improved early initiation of breastfeeding appears to be key in predicting neonatal mortality [3]. In the world sixty percent of the infant and young child deaths occur due to malnutrition where two-thirds of these deaths attributed to sub-optimal child feeding practices and infectious disease [4]. The impacts of inappropriate infant feeding practice are great in developing countries [5].

The beneficial effects of breastfeeding children are decrease risk for ear and respiratory infections, atopic dermatitis, gastroenteritis, necrotizing enter colitis, type 2 diabetes, and 


\section{Indo Global Journal of Pharmaceutical Sciences, 2017; 7(2): 134-142}

sudden infant death syndrome. For mothers it decreased risk of breast and ovarian cancer, and type 2 diabetes and speeding the return of uterine tone, stopping post-birth bleeding, and temporarily suppressing ovulation, which aids the spacing of children [6]. WHO and UNICEF recommend that mothers put newborns to the breast within one hour of birth, breastfeed infants exclusively for the first six months and continue to breastfeed for two [7].

In Ethiopia breastfeeding is nearly universal. $96 \%$ of children have been breastfed during some period in their lives and this varies minimally across regions. Although $\mathrm{BF}$ is universal, appropriate breastfeeding practices are not always followed. Timely feeding practice tops the table of life-saving interventions for new-borns $[8,9]$.

Breastfeeding practice is a vital component of primary health care. The government has been implementing the Babyfriendly Hospital Initiative (BFHI) and the community integrated management of childhood illnesses (IMCI) program $[10,11]$

\section{MATERIALS AND METHODS}

The study was conducted in Benishangul Gumuz Regional State, North West Ethiopia. The Region consists of three zones (Assosa, Metekele and Kamashi), one special woreda (Maokomo) and Assosa city administration. Based on the data from regional health bureau the total population of the region is 993,584. Benishangul Gumuz Regional state consists 36 health centers, 398 health post, and 2 hospitals. Beside this the region also has one University located in Assosa the capital city of the region which is located 661 kilometres (k.ms) Northwest of Addis Ababa and one Health science college in Pawe woreda. The study was conducted in Bullen, Mandura, Assosa, Homosha, Kamashi, Bello And Maokomo woreda on atotal of 46 roddomly selected kebeles from April 20$29 / 2015$

A community-based cross-sectional study supplemented by qualitative study was used.

For quantitative study: All infant paired mothers living in BG Region.

For qualitative study: All infant paired mothers, community and religious leaders in the region.

For the Quantitative method: All infant paired mothers randomly selected woreda and kebele.

For qualitative study: All infant paired mothers, community and religious leaders randomly selected from each kebele.
Since the study subjects are mother-infant paired groups, mothers whose infant age less than two year were included as study population. Mothers with infants who are seriously ill and unable to communicate from any cause were excluded from the study.

The quantitative sample size for this particular study will be calculated using formula for a single population proportion considering the following assumptions

$$
\frac{\mathrm{n}=\left(\mathrm{Z}_{\alpha / 2}\right)^{2} \mathrm{p}(1-\mathrm{p})}{\mathrm{d}^{2}}
$$

Where: $\mathrm{n}=$ required sample sizes

$\mathrm{Z}_{\alpha / 2}=$ critical value for normal distribution at $95 \%$ confidence level which equals to 1.96

$\mathrm{P}=(42.2 \%)$ prevalence of early initiation of breastfeeding of BGRS, EDHS 2011. $\mathrm{d}=$ an absolute precision (margin of error 5\%). Using the above formula

$$
\mathrm{n}=\frac{(1.96)^{2}}{(0.422(1-0.422)}
$$

$\mathrm{n}=375$ mothers with infants

Using Design Effect of 1.5 then sample size become 562 .

Adding 5\% of non-respondent the final sample becomes 590 .

10 focused group discussions (FGDs) on motherinfant/child pair and 20 in-depth interviews on community and religious leaders were conducted until the saturation of idea reached in all purposively selected woreda.

There are 20 woreda and one administrative town in Benishangul Gumuz Regional state. The sampling procedure of this study was started by using classification of the 20 woreda and one administrative town into strata since population of each woreda is known to have heterogeneity with regard to infant and child feeding practice. The total populations size of infant-paired mothers with age less than two years found in each woreda were calculated using the conversion factors and again proportionally allocated sample size for each woreda were reallocated to their respective urban and rural kebele proportionally. Finally the total of 788 study participant were selected using simple random sampling methods for this purpose health extension workers were assisted the data collectors as a guider in locating where mother-infants pairs were found.

The quality of the data was assured through careful design, translation, retranslation and pre-test of the questionnaire. The questionnaire was adopted in English from different literature and translated into Amharic and then back into English by experts. Pre-testing was done 10 days prior to actual data collection nearby Kebele. The participants in this sample pretest kebele were not included in the study. 


\section{Indo Global Journal of Pharmaceutical Sciences, 2017; 7(2): 134-142}

Data collectors and supervisors were trained for one day. The principal investigator and the supervisors were checked the collected data for completeness and corrective measures were taken accordingly. The collected data were cleaned, coded and explored before.

Data were collected using semi-structured and interviewer guided questionnaire adopted from related studies and EDHS. The questionnaire adopted were modified and contextualized to the local situation and the research objective after pre testing. Open-ended questionnaire were also used for Focused Group Discussion and in-depth interview.

Health care providers (who understand, speak and write the local language) were used as data collectors. They were trained for one day by the principal investigator on the objective of the study, data collection tools and sampling procedures.

Before the actual data collection, the questionnaire was pretested on a similar kebele which were not included in the study. Amendments on the questionnaire were made accordingly after the pre-test.

After identifying the study subjects with random selection infant paired mother, face to face interview data collection method was employed. To maximize the data quality obtained by the use of semi- structured questionnaires FGDs were undertaken in group of infant paired mother. Each FGD were consisting of minimum of six members. The members of each FGD were selected by principal investigator (Researcher) or supervisors. FGD were moderated by Principal investigators/supervisors and translator who able to speak, hear and write local languages.

Data, the data was checked for completeness and entered in to Epi-Info version of 3.1 then it was exported to SPSS version 20.0. Descriptive statistics was calculated to describe the overall distribution of the study subject with the variables under study. Bivariate and multivariable logistic regression analyses were used to determine the presence of statistically significant associations between outcome and the independent variables. The strength of association was measured by adjusted odds ratios and 95\% confidence intervals using multivariate analysis. Statistical significance were declared at $\mathrm{P}<0.05$. Finally the result was presented in tables and graphs.

For qualitative data, data was transcribed in to an English text by the principal investigator from the note and by replaying the tape recorder. The different ideas in the text were merged in their thematic areas and thematic framework analyses were employed to extract meanings out of the texts manually. Then finally results were presented in narratives in triangulation with quantitative data.

Socio-demographic and economic characteristics: (age, sex, marital status, residence, occupation, educational status, ethnicity, religion, and monthly income). Sociocultural factors: (provide butter for new born infants, discarding the first milk, giving water for new born before breast feed). Maternal related factors/practice: (use of ANC service, use PNC services, place of delivery, access to health care, access to health information, Knowledge). Obstetrics and Medical factors: (Breast illness, Mode of delivery, Gastroenteritis, respiratory infections). Ethical clearance was obtained from Ethical Review Committee OF Benishangul Gumuz Regional Health Bureau, and then submitted to both zonal and woreda health bureau where study is conducted. The study participants were informed about the purpose of the study and finally their oral consent were obtained before interview. They also notified that the information provided by each respondent was kept confidential with assurance of the right to refuse or terminate the interview at any point.

\section{RESULTS}

Socio-demographic Characteristic of the Respondents:

From a total of 788 study subjects, 770 respondents were provided accurate information with a response rate of $97.7 \%$. All of the respondents were between the age group of 16-40 years with the mean age of $25.5+5.2$ years. Among the study participants of infant paired mothers 391(50.8\%) were followers of Muslim while 261(33.9\%) were Christians, 111(14.4\%) were protestant. Regarding ethnic composition of the respondents $227(29.5 \%)$ were Berta, 142(18.4\%) were Gumuz, 66(8.6\%) was Shinasha and 37(4.8\%) respondents were Mao. Among the study participants of infant paired mothers about $629(81.7 \%)$ lives in rural while

141(18.3\%) lives in urban. Regarding marital status $711(92.3 \%)$ were married the $30(3.9 \%)$ were single. Among the respondent of mothers $247(32.1 \%)$ were housewives, $423(54.9 \%)$ were farmer. In case of educational status out of the study participants $381(49.5 \%)$ were unable to read and write, $103(13.4 \%)$ were learnt grade one to grade four and $37(4.8 \%)$ were completed grade ten.

\section{Obstetrics characteristics and knowledge of the respondents}

Among a total of study participants $691(89.7 \%)$ mothers had attended ANC for their last birth. 533(46.1\%) were delivered at health institution and 514(53.8\%) were delivered at home. 
Indo Global Journal of Pharmaceutical Sciences, 2017; 7(2): 134-142

Table 1:- Socio-demographic related variables of infant paired mothers $(\mathbf{n}=770)$ in Benshangul Gumuz regional state Northwest Ethiopia, 2016

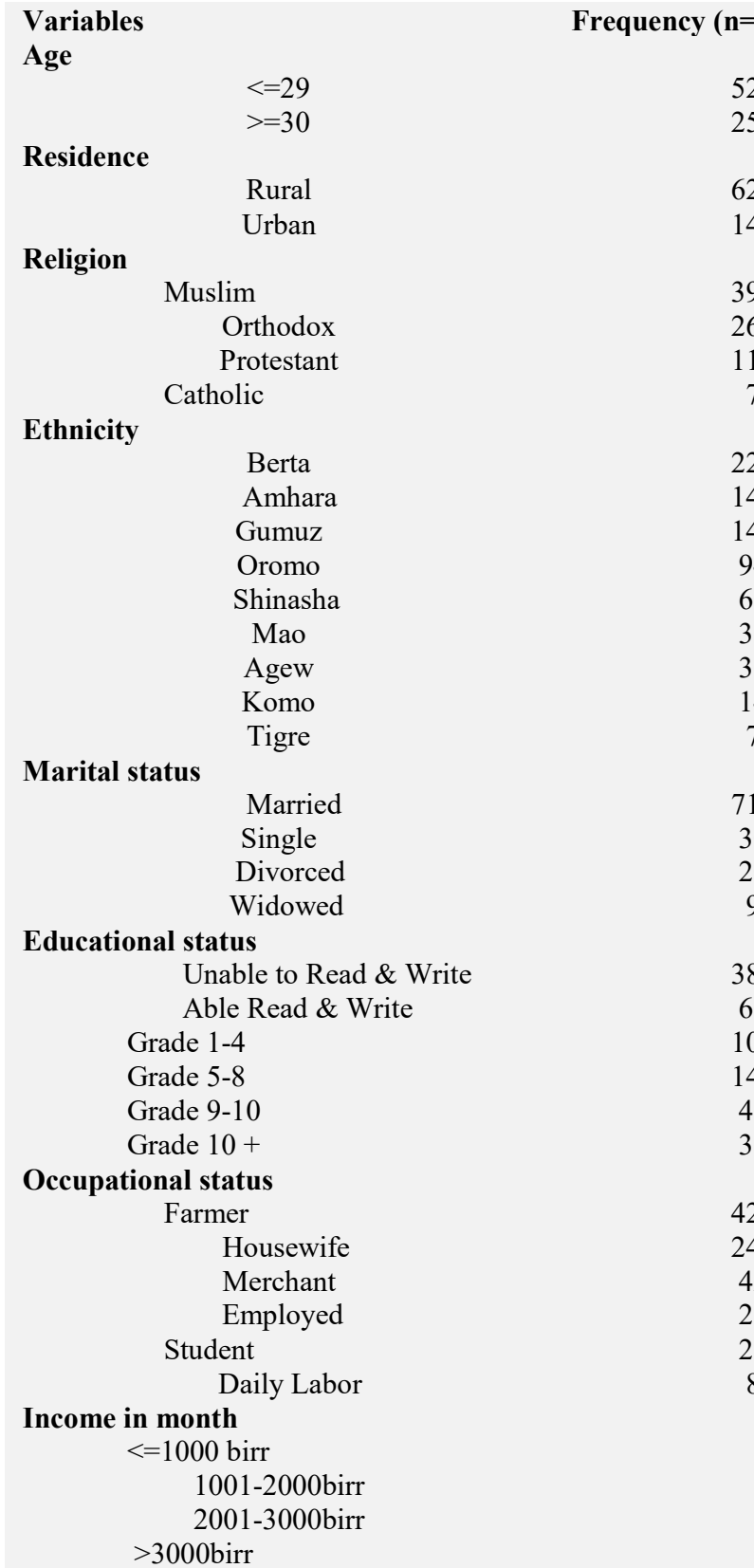

The proportions of mothers who assisted delivery at home by NTTBA, TTBA, alone, were $83(10.8 \%)$, and $46(6 \%)$ respectively

Five hundred (64.9) of the respondents were informed about breast feeding practice during ANC service. Of the informed mothers on breast feeding practice about 19(3.8\%) were informed only about early initiation of breast feeding and About sixty two percent of mothers were informed on (early initiation, exclusive breast feeding for six months, introduction

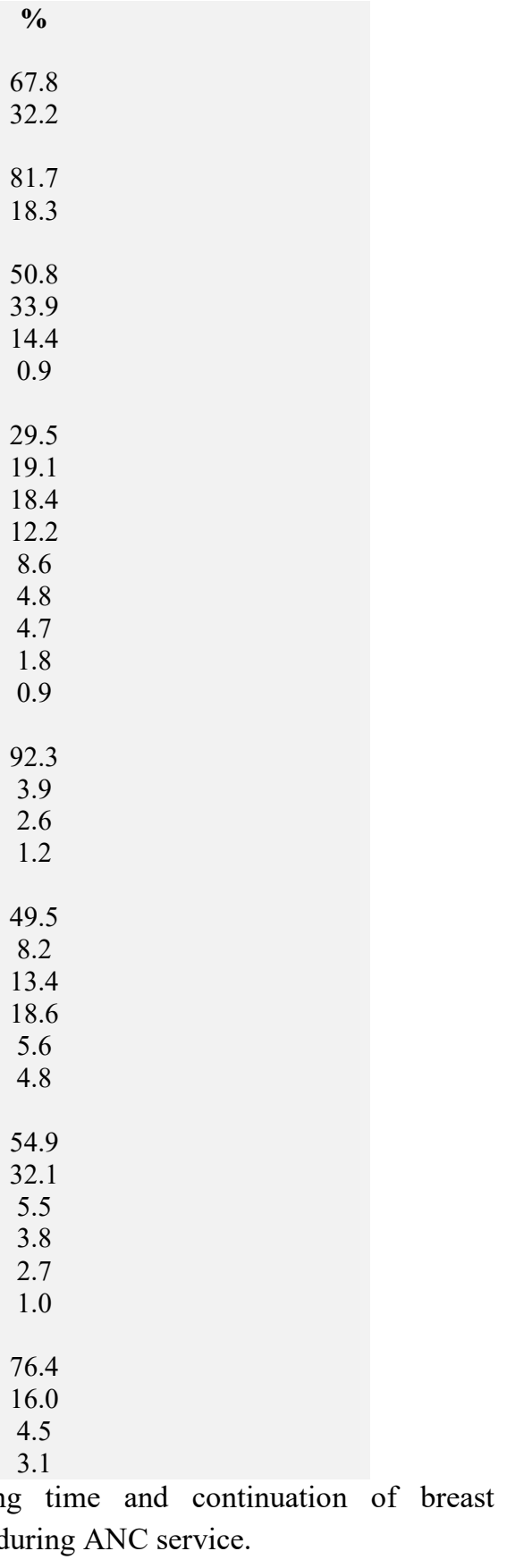

feeding for two years) during ANC service.

About 61 percent of mothers had adequate knowledge about function of breast feeding practice. Among a total of the study participants Mothers who had adequate knowledge about function early initiation of breast feeding practice and timely introduction of complementary feeding practice were $563(73.1 \%)$ and $707(91.8 \%)$ respectively. 
Indo Global Journal of Pharmaceutical Sciences, 2017; 7(2): 134-142

Table 2:- Obstetrics and knowledge related variables of infant paired mothers $(n=770)$ in Benishangul Gumuz regional state Northwest Ethiopia, 2016.

\begin{tabular}{|c|c|c|}
\hline \multirow{2}{*}{\multicolumn{3}{|c|}{$\begin{array}{l}\text { Variables } \\
\text { ANC follow up }\end{array}$}} \\
\hline & & \\
\hline No & 79 & 10.3 \\
\hline Yes & 691 & 89.7 \\
\hline \multicolumn{3}{|l|}{ Parity } \\
\hline Para One & 196 & 25.5 \\
\hline Para Two And Above & 574 & 74.5 \\
\hline \multicolumn{3}{|l|}{ Place of delivery } \\
\hline Home & 415 & 53.9 \\
\hline Institution & 355 & 46.1 \\
\hline \multicolumn{3}{|l|}{ Mode of delivery } \\
\hline Normal delivery & 747 & 97.0 \\
\hline Cesarean section & 20 & 2.6 \\
\hline Instrumental delivery & 3 & 0.4 \\
\hline \multicolumn{3}{|l|}{ Delivery assisted by } \\
\hline Health professional & 364 & 47.3 \\
\hline TTBA & 53 & 6.9 \\
\hline NTTBA & 83 & 10.8 \\
\hline Alone & 46 & 6.0 \\
\hline Family & 224 & 29.1 \\
\hline \multicolumn{3}{|c|}{ Informed about feeding practice during ANC follow up } \\
\hline No & 283 & 36.8 \\
\hline Yes & 487 & 63.2 \\
\hline \multicolumn{3}{|c|}{ Informed about feeding practice during PNC follow up } \\
\hline No & 270 & 35.1 \\
\hline Yes & 500 & 64.9 \\
\hline \multicolumn{3}{|l|}{ Knowledge about function of Breast milk } \\
\hline inadequate knowledge & 302 & 39.2 \\
\hline adequate knowledge & 468 & 60.8 \\
\hline \multicolumn{3}{|l|}{ Knowledge about early initiation Breast feeding } \\
\hline inadequate knowledge & 207 & 26.9 \\
\hline adequate knowledge & 268 & 73.1 \\
\hline \multicolumn{3}{|c|}{ Knowledge about timely introduction of complementary } \\
\hline inadequate knowledge & 63 & 8.2 \\
\hline adequate knowledge & 707 & 91.8 \\
\hline
\end{tabular}

Infant and young child breast feeding practice:

About $99.7 \%$ of the respondents were responds that infant breast feed their infants during data collection period. The proportion of early initiation of breast feeding practice in this research was 414(53.8\%). A total of $39(5.1 \%)$ mothers practice Prelacteal feeding (caw milk, sugar with water or $40 \%$, water, better were $14(36 \%), \quad 11(28.9 \%), \quad 8(21.1 \%) \quad$ and $\quad 5(12.2 \%)$ respectively.

About $5.8 \%$ of infants were breastfed exclusively for one month, $12.3 \%$ of infants were breastfed exclusively to 2-3 month s of age.

Factors associated with early initiation of breast feeding and timely Introduction of Complementary feeding practice:

From the bivariate analyses variables that fulfilled the minimum requirement $(0.2$ level of significance in this study) were entered in to multivariate logistic analysis. The multivariate logistic regression which controls the effect of confounding variables was used by taking all covariates into account simultaneously for early initiation of breast feeding and timely introduction of Complementary feeding practice. Multi-logistic regressions showed that the present parsimonious model adequately fits the data for timing introduction of Complementary feeding practice as P- value from Hosmer and Lemeshow test was 0.590 .

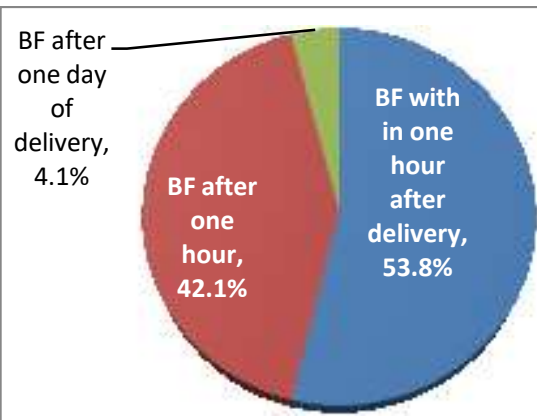

Figure 1:-Distribution of women by time of initiation of breast feeding practice 
Indo Global Journal of Pharmaceutical Sciences, 2017; 7(2): 134-142

After applying bivariate and multi-logistic regressions, four variables were found to be significantly associated with early initiation of breast feeding practice. These were who delivered at health institution $(\mathrm{AOR}=12.18)$, mothers who had ANC follow up (AOR=2.02), mothers who had adequate knowledge about breast feeding $(\mathrm{AOR}=2.02)$ and mothers who had adequate knowledge about early initiation of breast feeding practice $(\mathrm{AOR}=$ 2.09) were more likely to start breast feeding practice within one hour of delivery.

On the other hand residences, gender, educational status of mothers were not associated with early initiation of breast feeding practice. (The results of Bivariate and multivariate analysis were summarized in Table 3 below).
Qualitative result:

"Thematic frame work analysis" was used for sorting transcribed information, looking for patterns, similarities, differences or contradictions. The results of qualitative parts were summarized in three parts as follows.

Regarding the results of FGD and In-depth interview on breastfeeding practice all mothers those

participated in the FGD in the five woreda stated that breastfeeding is widely practiced in their residential area and they believe it is important for normal child development, strength and health. They also stated that timely introduction of complementary food on the top.

Table 3: Factors associated with early initiation of breast feeding practice $(n=770)$ in Benishangul Gumuz regional state Northwest Ethiopia, 2007 .E.C.

Explanatory Variable

Child sex

Male

Female

\section{Residence}

Rural

Urban

Educational level

Not Educated

Grade 1-4

Grade Five And Above

Place of delivery

Home

Institution

ANC follow up

no

yes

info about FDP during ANC

No

Yes

Knowledge about importance

of breast milk

inadequate knowledge 175

adequate knowledge

Knowledge about of early

initiation breast feeding

No

Yes

$135 \quad 72 \quad 1$

$225 \quad 338$

49

89

$304 \quad 387$

205

282
Early initiation of breast feeding practice

No Yes Crude OR(95\% CI) P-value Adjusted OR(95\% CI)

$199 \quad 211 \quad 0.86(0.65-1.14)$

$161 \quad 199 \quad 1$

$311 \quad 321 \quad 1$

$1.76(1.20-2.58)^{*}$

$\begin{array}{lll}198 & 183 & 1\end{array}$

$\begin{array}{lll}76 & 90 & \mathbf{1 . 2 8}(\mathbf{0 . 8 9}-\mathbf{1 . 8 5})\end{array}$

$86 \quad 137 \quad \mathbf{1 . 7 2 ( 1 . 2 3 - 2 . 4 1 ) *}$

$\begin{array}{lll}300 & 115 & 1\end{array}$

$60 \quad 295 \quad \mathbf{1 2 . 8 5 ( 9 . 0 9 - 1 8 - 2 2 ) *}$

$56 \quad 23 \quad 1$

$3.10(1.54-5.15)^{*}$

$\begin{array}{lll}155 & 128 & 1\end{array}$

$1.67(1.24-2.24)^{*}$

$127 \quad 1$

$185283 \quad 2.22(\mathbf{1 . 5 7}-\mathbf{2}, \mathbf{8 3}) *$

$0.05 \quad 1$

$1.9(1,00-2.23)^{* * *}$

$1.023(0.65-1.60)$

$0.935(0.615-1.42)$

0.001

12.18(8.29-17.98)**

$0.03 \quad 1$

$2.02(1.07-3.92)^{* *}$

$0.22 \quad 1$

$0.69(0.38-1.25)$

0.00

2.08(1.28-3.39)** 


\section{Indo Global Journal of Pharmaceutical Sciences, 2017; 7(2): 134-142}

Timely initiation of breast feeding:- although all mothers of the five woreda expressed their belief breast milk is good for children making them strong and health, Most of them did not breastfed within an hour after delivery. As most of them stated early initiation of breastfeeding practice within one hour depends on several factors like place of delivery, mode of delivery, health status of the mothers and the child and knowledge of the mothers. Still in some kebele of each woreda there are a promising awareness creation done on this issue.

As one of the mothers from (Abrhamo kebele, Assosa woreda) state as:

"All my child born before him has not been given a breast within an hour because I gave all of birth at home and I consider the first milk is not clean enough for them to be fed but after we have got an education from health workers and HEW in house-to- house mode I gave a birth of my young child at health center and immediately I put my breast in to his mouth."

\section{DISCUSSION}

Ninety-nine percent of mothers had ever practiced breastfeeding which is almost similar to the national ever breastfeeding rate $(96 \%)$. This study showed that the prevalence of early initiation of breast feeding practice within one hour of delivery was 414(53.8\%). This finding is lower as compared to research conducted in Kapasia Bangladesh 67\% [19], other Ethiopian region like in Sidama zone 80.1\% [21], Wolayita zone 90\% [22], Bahirdar city administration $87 \%$ [24], Mekelle 77\% [23], Debrebrhan 84.5\% [26] and Tiyo Arsi zone $67.3 \%$ [27] and the national early initiation of breast feeding prevalence in Ethiopia which was (92\%). Such variations may due to methodological variations between studies, dissimilarities in infant and maternal sociodemographic characteristics and other differences in sociocultural, economical, health and health service utilization characteristics between respondents of the referenced areas and the study place.

In this study mothers who had ANC visit of their last child were about two times more likely initiate early breast feed as compared to counterpart $[\mathrm{AOR}=2.02(1.07-3.92)]$. This is in line with study conducted in Mekelle [23] and Debrebrhan [26]. This could be those who have ANC follow up have the possibility being aware of early initiation of Breast feeding practice during follow up and adequate knowledge on breast feeding tend to introduce feedings in early as possible.
Those mothers who were knowledgeable on correct time of breastfeeding initiation were two times more likely to practice early initiation than those who were not knowledgeable 2.08(1.28-3.39). This implies the importance of maternal knowledge on correct initiation time in improving early initiation of breastfeeding this is also in line with research conducted in Bahirdar [24].

Those mothers who delivered current child at health institution were about twelve times more likely to initiate breastfeeding early than those mothers who delivered at home 12.18(8.29-17.98) . This finding was consistent with those of other studies in Mekelle [23], east haraghe zone [29], Nairobi Kenya [28]. This might be due to the fact that health institution delivery is the best source of information for early initiation of breastfeeding.

The qualitative report of the study showed that Most of them did not breastfed within an hour after delivery of a baby as most of them stated early initiation of breastfeeding practice within one hour depends on several factors like place of delivery, mode of delivery, health status of the mothers and the child and knowledge of the mothers.

Most of them stated that early initiation of breastfeeding practice within one hour depends on several factors like place of delivery, mode of delivery, health status of the mothers and the child and knowledge of the mothers.

As one of the mother from (Abrhamo kebele, Assosa woreda) stated that:- "for all my children I did not gave breast milk within one hour of my delivery because I gave all birth at home and I consider the first milk is not clean enough to fed."

\section{CONCLUSION}

Early initiation of breast feeding is low as nearly half the mothers did not start breastfeeding with one hour after delivery and still lower than the national plan. ANC follow up, place of delivery, having adequate knowledge, were the contributing factors early initiation of breast feeding. Increasing maternal knowledge on early initiation of breast feeding by educating the 
mothers both at community and institutional levels and enhance adequate uterine contraction.

\author{
ABBREVIATIONS \\ ANC: Antenatal care \\ BF: Breastfeeding \\ EBF: Exclusive breastfeeding \\ EDHS: Ethiopian Demographic and Health \\ Survey \\ IYCF: Infant and Young Child Feeding \\ WHO: World Health Organization.
}

\section{CONFLICTS OF INTEREST}

The author declares that there is no conflict of interests regarding the publication of this paper.

\section{AUTHOR'S CONTRIBUTION}

Yonas Deressa and Girma Tadese designed the study, performed the statistical analysis, and drafted the paper. Muluwas Amentie reviewed and advised the whole research process

\section{ACKNOWLEDGEMENTS}

The authors very grateful to the Benishangul Gumuz Regional Health Bureau for the genuine collaboration to undertake this research in the region and would like to thank all mothers who participated in this study for their commitment in responding to interviews.

\section{REFERENCES}

[1]. Jones G, Steketee R, Black R, Bhutta Z, Morris S. How many child deaths can we prevent this year? Lancet 2003; 362: 65-71.

[2]. Darmstadt GL, Bhutta Z, Hasan B, Haws R. Evidencebased, cost-effective interventions: how many newborn babies can we save? Lancet 2005; 365: 97788

[3]. Edmond KM, Zandoh C, Quigley MA, Amenga-Etego S, Owusu-Agyei S, Kirkwood BR. Breastfeeding initiation increases risk of neonatal mortality. Pediatrics 2006; 117: 380-6.

[4]. UNICEF. Global Strategy for Infant and Young Child Feeding, Geneva, Switzerland. 2003.

[5]. WHO. Report of the global consultation on Summary of guiding principles for complementary feeding of the breastfed child, Geneva Switzerland. 2001

[6]. CDC, Division of Nutrition and Physical Activity 2007. Does breast feeding reduce the risk of pediatric overweight. Research to Practice Series, No. 4.
[7]. World Health Organizations. Global Strategy for Infant and Young Child Feeding. World Health Organization; Geneva. 2003.

[8]. Central Statistical Agency [Ethiopia] and ORC Macro 2006.

[9]. EDHS 2005,

[10]. Miller NP, Amouzou A, Tafesse M, Hazel E, Legesse H, Degefie T, Victora CG, Black RE, Bryce J. Integrated community case management of childhood illness in Ethiopia: implementation strength and quality of care. Am J Trop Med Hyg. 2014;91(2):424-34.

[11].Labbok MH. Global baby-friendly hospital initiative monitoring data: update and discussion. Breastfeed Med. 2012;7:210-22.

[12].WHO/UNICEF. Global Strategy for Infant and Young Child Feeding. 2006.

[13]. Central Statistical Agency. Ethiopia Demographic and Health Survey 2011 A.A, Ethiopia. Calverton: ICF International; 2012.].

[14]. Ogbo FA, Page A, Idoko J, Claudio F, Agho KE. Diarrhoea and suboptimal feeding practices in nigeria: evidence from the national household surveys. Paediatr Perinat Epidemiol. 2016;30(4):346-55

[15].Kolola T, Ekubay M, Tesfa E, MorkaW. Determinants of Neonatal Mortality in North Shoa Zone, Amhara Regional State, Ethiopia. PLoS ONE. 2016: 11(10): e0164472. doi:10.1371/journal

[16].Setegn T, Gerbaba M, Belachew T. Determinants of timely initiation of breastfeeding among mothers in Goba Woreda, South East Ethiopia: A cross sectional study. BMC Public Health. 2011;11:217.

[17]. Sharma A et al. Factors associated with early initiation of breastfeeding among mothers of tribal area of Madhya Pradesh, India: a community based cross sectional study. Int J Community Med Public Health. 2016; 3(1):194-199

[18].Dyah Ayu Inayati, Veronika Scherbau m, Ratna Chrism iari Purwestri, Elizabeth Hormann, Nia Novita Wirawan, Julia Surya ntan etal. Infant feeding practices among mildly wasted children: a retrospective study on Nias Island, Indonesia. International Breastfeeding Journal 2012, 7:3.

[19]. Sultana S, Hoque A, Saleh F. Infant and Young Childfeeding Practices and their Nutritional Status in a National Nutrition Programme Area in Bangladesh: A Cross-Sectional Study. J Hum Nutr Food Sci. 2014, 2(2): 1028.

[20].Community-Based Sub-Component of Ethiopian National Nutrition Program, addiss ababa Ethiopia; Addis Continental Institute of Public Health, 2009.

[21].Bernadette P. Marriott, Alan White, Louise Hadden, Jayne C. Davies, John C. Wallingford. World Health Organization (WHO) infant and young child feeding indicators: associations with growth measures in 14 low-income countries. Blackwell Publishing Ltd Maternal and Child Nutrition, 2011.

[22].Regassa N. Infant and child feeding practices among farming communities in Southern Ethiopia. Kontakt 2014; 16(4): e215-e222; http://dx.doi.org/10.1016/j.kontakt.2014.09.002

[23]. Berhe et al. Determinants of Breast Feeding Practices among Mothers Attending Public Health Facilities, 


\section{Indo Global Journal of Pharmaceutical Sciences, 2017; 7(2): 134-142}

Mekelle, Northern Ethiopia; a Cross Sectional Study. IJPSR, 2013; 4(2): 650-660.

[24]. Abdulbasit, Musa Seid. Vaginal Delivery and Maternal Knowledge on Correct Breastfeeding Initiation Time as Predictors of Early Breastfeeding Initiation: Lesson from a Community-Based Cross-Sectional Study. Hindawi Publishing Corporation. 2014, Article, ID $904609,6$.

[25].Legesse et al. Prelacteal feeding practices and associated factors among mothers of children aged less than 24 months in Raya Kobo district, North Eastern Ethiopia: a cross-sectional study. International Breastfeeding Journal (2014) 9:189.

[26].Gultie and Sebsibie. Determinants of suboptimal breastfeeding practice in Debre Berhan town, Ethiopia: a cross sectional study. International Breastfeeding Journal (2016) 11:5.

[27]. Woldemichael B, Kibie Y (2016) Timely Initiation of Breastfeeding and Its Associated Factors among
Mothers in Tiyo Woreda, Arsi Zone, Ethiopia: A Community- Based Cross Sectional Study. Clinics Mother Child Health 13: 221. doi:10.4172/20907214.1000221.

[28].Elizabeth W Kimani-Murage, Nyovani J Madise, JeanChristophe Fotso, Catherine Kyobu tungi, Martin K Mutua, Tabither M Gitau, Nelly Yatich. Patterns and determinants of breastfeeding and complementary feeding practices in urban informal settlements, Nairobi Kenya. BMC Public Health 2011, 11:396.

[29].Kibebew Abera. Infant and Young Child Feeding Practices Among. Mothers Living in Harar, Ethiopia. Harar Bulletin of Health Sciences. 2012,4.

[30].Tilahun Tewabe. Timely initiation of breastfeeding and associated factors among mothers in Motta town, East Gojjam zone, Amhara regional state, Ethiopia, 2015: a cross-sectional study. BMC Pregnancy and Childbirth, 2016; 16(1): 314.

Indo Global Journal of Pharmaceutical Sciences( ISSN 22491023 ; UGC Journal No.: 44477; CODEN- IGJPAI; NLM ID: 101610675) indexed and abstracted in EMBASE(Elsevier), UGC Journal List, National Library of Medicine (NLM) Catalog, Elsevier( EMBASE), ResearchGate, Publons, CAS (ACS), Index Copernicus, Google Scholar and many more. For further details, visit http://iglobaliournal.com 